\title{
Alternativas de desarrollo: viejos desafíos y nuevas hibridaciones en China y América Latina
}

\author{
Paul Bowles* \\ Henry Veltmeyer**
}

Resumen. Para la corriente dominante de los Estudios de Desarrollo Internacional, el «desarrollo» supone la emancipación de la pobreza para más de mil millones de personas que no pueden satisfacer sus necesidades humanas básicas, en un mundo que nunca ha sido más rico en términos materiales. Los estudios críticos de desarrollo pretenden analizar por qué los proyectos de desarrollo implementados no han logrado realizar su proclama emancipatoria tras cinco décadas de múltiples iniciativas y recursos, y propone alternativas al modelo convencional. Para ello, propone examinar y teorizar la dinámica de desarrollo del sistema capitalista; formular herramientas para analizar a los Estados, sociedades y comunidades que han tratado de generar mejores condiciones de vidas y desafían los modelos ortodoxos; articular la resistencia al capitalismo y la búsqueda de alternativas en las periferias; concebir el subdesarrollo o desarrollo capitalista periférico y el poscapitalismo o posdesarrollo a partir del desarrollo desigual; evidenciar el fracaso de la teoría y política del desarrollo económico dominante porque no comprende o ignora la dinámica subyacente del desarrollo capitalista. Por lo anterior se plantea ir más allá del capitalismo a partir de las fuerzas del cambio progresistas y orientadas por un desarrollo alternativo. Se analizan lo casos de China y América Latina, donde se detectan una serie de hibridaciones que pueden ofrecer enseñanzas sobre cómo pueden evolucionar las alternativas al capitalismo.

Palabras clave: capitalismo, estudios críticos del desarrollo, hibridación, China, América Latina.

* Profesor de Economía y Estudios Internacionales en la Universidad del Norte de Columbia Británica, Canadá.

** Docente investigador de la Unidad Académica en Estudios del Desarrollo de la Universidad Autónoma de Zacatecas, México.

Traducción del inglés por Humberto Márquez Covarrubias. 


\section{Development alternatives: old challenges and new hybridization in China and Latin America}

Abstract. In the current, dominant conceptualisation of International Development Studies, «development» is based on the emancipation from poverty for the more than one-billion people who are unable to satisfy their basic human needs, in a world that has never been richer in material terms. Critical development studies attempts to understand why the many projects of development that have been implemented have not led to the stated emancipatory goal after five decades of multiple initiatives and resources, and propose alternatives to the conventional model. To that end, this article examines and theorizes the dynamic of capitalist system's development project; offers tools for the analysis of States, societies and communities which have attempted to create better living conditions and defy orthodox models; outline the resistance to capitalism and the search for alternatives in the peripheries; conceptualize peripheral capitalist subdevelopment or development and post-capitalism or post-development from a perspective of unequal development; reveal the failure of dominant economic development theory and policy that is unable to understand or ignores the underlying dynamic of capitalist development. Consequently, the article proposes going beyond capitalism with the forces of progressive change, oriented toward an alternative development. It analyzes case studies in China and Latin America, where a series of hybridizations are identified that could offer lessons for how to create alternatives to capitalism.

Keywords: capitalism, critical development studies, hybridization, China, Latin America. 
De acuerdo con sus defensores institucionales y profesionales de la corriente principal de los estudios internacionales de desarrollo, el «desarrollo» hace al menos un reclamo emancipatorio importante —emancipación de la pobreza para más de mil millones de personas (exactamente cuántos depende de cómo se define y cuenta la pobreza) que persiguen su vida cotidiana en condiciones de pobreza (entendida como la incapacidad de las personas para satisfacer sus necesidades humanas básicas) en un mundo que nunca ha sido más rico en términos materiales. El Banco Mundial (BM), por ejemplo, en su cabecera describe que sus objetivos son «acabar con la pobreza extrema dentro de una generación e impulsar la prosperidad compartida» (BM, s/f). Se entiende que esto último significa aumentar los ingresos de 40\% inferior de la población en cada país; de ahí el interés del BM en el crecimiento/desarrollo «favorable a los pobres» e «inclusivo». Por su parte, el Programa de las Naciones Unidas para el Desarrollo (PNUD), en su concepción del desarrollo humano y sus objetivos de desarrollo sostenible, y en su «llamado universal a la acción para acabar con la pobreza, proteger el planeta y garantizar que todas las personas disfruten de la paz y la prosperidad» (PNUD, s/f), define el desarrollo de manera más amplia, pero la reducción de la pobreza permanece como una estrategia central.

Los estudios críticos de desarrollo (ECD) son una rama de los estudios de desarrollo que busca examinar por qué, a pesar de más de cinco décadas de esfuerzos concertados y recursos dedicados, las audaces afirmaciones emancipatorias hechas para el desarrollo no se han realizado y cómo se deben encontrar alternativas al modelo convencional. En este sentido, los ECD se basan en una serie de entendimientos clave.

El primero es que es esencial examinar de cerca y teorizar la dinámica de desarrollo del sistema operativo subyacente: el capitalismo. En la corriente 
principal de los estudios del desarrollo, la suposición asumida de que el capitalismo proporciona el mejor, si no es que el único, sistema para lograr el objetivo emancipatorio fundamental del desarrollo ha llevado a la desaparición virtual de cualquier mención, y mucho menos de la teorización, del capitalismo como sistema. Sin embargo, la producción de pobreza está asociada y es el resultado directo de una multiplicidad de fuerzas como el colonialismo, el imperialismo, la guerra, el racismo, el patriarcado y la destrucción ecológica que no pueden entenderse ni superarse completamente sin una comprensión clara de la dinámica y las contradicciones fundamentales del capitalismo. En consecuencia, los ECD se preocupan y ofrecen mucho más que la simple emancipación de la pobreza, es decir, la emancipación del capitalismo. Si bien ha habido casos previos de «crisis en el desarrollo» $\mathrm{o}$ «impases en el desarrollo», la coyuntura actual nos lleva a argumentar que, desde una perspectiva de los ECD, la crisis del desarrollo no puede separarse de las crisis del capitalismo.

En segundo lugar, el «desarrollo» visto a través de lentes críticos — ECD— proporciona un terreno rico y herramientas útiles para examinar cómo los estados, las sociedades y las comunidades han tratado de construir mejores vidas para sus miembros mientras desafían los modelos ortodoxos prevalecientes y extraen lecciones de estas experiencias. En estas experiencias, podemos ver mejor el potencial y las dificultades de las diferentes vías e ir más allá del capitalismo. Este análisis histórico también nos permite ir más allá del enfoque a menudo eurocéntrico de gran parte del debate sobre el poscapitalismo y el neoliberalismo autoritario que, de hecho, se probó en la periferia, por ejemplo, en Chile en la década de 1970, antes de su manifestación populista actual en partes del centro.

En tercer lugar, los ECD toman como axiomático que la resistencia al avance del capital en el proceso de desarrollo y la búsqueda de alternativas 
al capitalismo pueden articularse, avanzar e implementarse en la periferia del sistema capitalista mundial. Durante gran parte del siglo XX, este fue el caso a medida que las revoluciones socialistas estatales, que comenzaron en Rusia en 1917, se extendieron a través de la periferia del sistema desde China, Cuba, Vietnam y Angola, por ejemplo. De hecho, la resistencia al capital prevaleció en gran parte de la periferia, aunque su mezcla con luchas anticoloniales y antiimperialistas significó que tomara diferentes formas en diferentes lugares. En cualquier caso, la periferia no ha sido ajena a la búsqueda de alternativas al capitalismo, sino más bien todo lo contrario.

En cuarto lugar, el desarrollo de las fuerzas productivas y las condiciones sociales asociadas de las personas en los países y las macrorregiones en todo el sistema mundial son muy desiguales, lo que nos permite concebir el subdesarrollo o el desarrollo capitalista periférico en algunos casos y el poscapitalismo o posdesarrollo en otros. Este concepto de desarrollo capitalista desigual es fundamental para el marxismo. Lo que agregan los ECD es una preocupación fundamental sobre las condiciones específicas del capitalismo en la periferia de lo que ha surgido como un sistema mundial. Por ejemplo, los marxistas han teorizado durante mucho tiempo que el desarrollo capitalista de la industria se basa en el desarrollo capitalista de la agricultura y un proceso asociado de proletarización — la transformación de un campesinado de pequeños productores agrícolas en un proletariado industrial y la creación de un ejército industrial de reserva de trabajo excedente-; sin embargo, no han podido entender lo que esto ha significado para el capitalismo en la periferia del sistema, a saber, la formación de un semiproletariado de trabajadores rurales sin tierra empobrecidos que en la década de 1990 constituirían la base social primaria de la resistencia al capitalismo bajo la forma de globalización neoliberal (Petras y Veltmeyer, 2013). 
Otro fracaso del marxismo tradicional, centrado como estaba en la construcción de una teoría de las leyes fundamentales del desarrollo capitalista, fue la incapacidad de apreciar la magnitud y el significado del avance de la búsqueda de recursos (resource-seeking) del «capitalismo extractivo» en la periferia del sistema (Veltmeyer y Petras, 2014). Los analistas que operan dentro de un marco de ECD, que combina la economía política con la ecología social, han determinado que las contradicciones del capital extractivo, combinadas con las contradicciones y las fuerzas de cambio generadas por la relación capital-trabajo y la relación centro-periferia — posiblemente las principales contradicciones del capitalismo - subyacen y explican el surgimiento de las fuerzas fundamentales y más poderosas del desarrollo capitalista y la resistencia organizada al capitalismo actual. Esto nos permite no sólo teorizar sobre la dinámica fundamental del desarrollo capitalista - la forma actual adoptada por el capitalismo como sistema mundial — sino también identificar las fuerzas de resistencia más poderosas, que en el contexto latinoamericano apuntan hacia varios modelos y vías de desarrollo alternativo.

En quinto lugar, la teoría del desarrollo económico dominante, cuyos parámetros son ejemplificados institucionalmente por el BM, el PNUD y la Comisión Económica para América Latina y el Caribe (Cepal), han seguido durante seis décadas diversas estrategias diseñadas para lograr un cambio y desarrollo social transformadores (mejora en la condición social de diversas poblaciones objetivo) basados en el supuesto de la necesidad de una reforma institucional y política. Ninguna de estas estrategias ha funcionado, porque las diversas teorías y prescripciones de políticas presentadas por estas instituciones no comprenden o ignoran la dinámica sistémica subyacente del desarrollo capitalista. Al mismo tiempo, mientras que algunos economistas 
políticos marxistas (Foster, 2002; Foster, Clark y York, 2010) han identificado las raíces de la crisis fundamental que ha acosado al sistema capitalista, y que, según algunos, eventualmente conducirá a su colapso o derrocamiento, y han aclarado la forma de todo el sistema tomada por las fuerzas del desarrollo capitalista, han fallado singularmente en identificar las fuerzas antisistémicas que están conduciendo o tienen el potencial de transformarnos o movernos más allá del capitalismo. Ni la economía del desarrollo ni la economía política marxista han servido para identificar las fuerzas que tienen el potencial de un cambio sistémico. Para esto, se argumenta, podríamos recurrir a los ECD.

\section{Ir más allá del capitalismo: fuerzas de cambio progresista y desarrollo alternativo}

El capitalismo es un sistema en crisis: una crisis multifacética y multidimensional con profundas raíces en el funcionamiento normal del sistema capitalista. La experiencia en las últimas tres décadas dentro del marco institucional y político de lo que en la década de 1980 se describió como el «nuevo orden mundial» —un sistema diseñado para liberar a las «fuerzas de la libertad económica» (el mercado, la empresa privada, el capital) de las restricciones regulatorias del Estado intervencionista - ha dejado este hecho claramente patente. En este contexto, el paradigma de los ECD ha surgido como un campo heterogéneo de estudios emancipatorios, un conjunto diverso de ideas de desarrollo alternativo y alternativas al desarrollo, en respuesta a las condiciones cambiantes del desarrollo capitalista y como un desafío intelectual a la ideología de la globalización neoliberal 
y los enfoques convencionales del «desarrollo». El rango de contribuciones a los ECD es extenso y ha sido revisado en otros lugares (véase Veltmeyer y Bowles, 2018; Veltmeyer y Delgado, 2018). En este artículo complementamos ese trabajo enfocándonos en la experiencia del desarrollo capitalista en dos macrorregiones (una de ellas un país y la otra una amalgama de unos 30 países), para analizar las implicaciones, posibilidades y vías para ir más allá del capitalismo. Nos centramos en estos dos casos de desarrollo capitalista no por sus supuestos «éxitos» o que ocasionen «esperanza» para un futuro mejor, sino por su importancia. En ambos, encontramos los desafíos actuales de para quién debería ser el «desarrollo» y qué constituye el «desarrollo», de cuál podría y debería ser el papel del Estado, qué relaciones de propiedad no capitalistas o poscapitalistas podrían ser posibles, e identificar las fuerzas de resistencia y de aquellos que bloquean la transformación social.

China, como la segunda economía más grande del mundo, ha sido testigo de décadas de rápido crecimiento económico sostenido y tiene un papel y una influencia globales cada vez más importantes en el pensamiento sobre el desarrollo en otras partes de la periferia. Como sólo el segundo país no occidental en desafiar el dominio occidental del sistema global desde la Revolución Industrial, y como el único que no forma parte de la alianza de seguridad occidental, ocupa un lugar único en los modelos y prácticas dominantes potencialmente desafiantes. Por ejemplo, su ejercicio de «poder blando» y su influencia financiera, como lo demuestra la Iniciativa del Cinturón y Ruta de la Seda, significa que su influencia en la configuración del desarrollo del siglo XXI en la periferia no puede ser ignorada. América Latina, por otro lado, históricamente una región de ricas contribuciones al pensamiento de desarrollo alternativo desde la teoría 
de la dependencia en adelante (Kay, 2018), en los últimos años ha servido como un laboratorio virtual de experimentos con modelos de desarrollo alternativo - una alternativa al avance del capital en el proceso de desarrollo, alternativas a las poderosas fuerzas del desarrollo capitalista. Estos incluyen la «marea rosa» de una década de regímenes progresistas, ya que varios gobiernos de la región buscaron avanzar en una forma más inclusiva de desarrollo (capitalista) o ir más allá del capitalismo, promoviendo nuevas formas de pensar como las encapsuladas por la noción de Buen Vivir/Vivir Bien (en solidaridad social y armonía con la naturaleza), una noción institucionalizada en las Constituciones (si no en la práctica política) de Bolivia y Ecuador (Acosta, 2012; Gudynas, 2011). Y la búsqueda de formas y modelos de desarrollo alternativos, también incluyó la construcción de una «economía social y solidaria» basada en el cooperativismo, la autogestión de los trabajadores y el desarrollo local, así como el intento de construir un «socialismo del siglo XXI» en Venezuela (Azzellini, 2017). A este respecto, analizamos cómo las experiencias de China y América Latina están dando forma al pensamiento sobre qué opciones para el cambio transformador en áreas periféricas en el futuro podrían ser posibles o factibles.

En nuestro análisis no nos centramos en las posibilidades de un cambio transformador repentino y revolucionario y el colapso repentino del capitalismo y su reemplazo con un plan detallado para una alternativa, sino en las perspectivas de cambio que no dependen del «catastrofismo» como punto de partida. Más bien nos centramos en las vías y la existencia de hibridaciones como formas institucionales de transición. Como argumentaremos, están surgiendo hibrididades que difuminan y rompen los límites entre el capitalismo y el poscapitalismo. 
Llegamos a la conclusión de que muchas de estas hibridaciones pueden no ser deseables desde una perspectiva emancipadora y contienen contradicciones problemáticas. Si bien puede haber islas de esperanza en el océano capitalista, la lección general extraída de nuestros estudios es que las perspectivas de una transición a una forma de desarrollo emancipador en la periferia en las condiciones actuales son bajas. Aun así, las formas y fuerzas de resistencia generalizadas y diversas que están surgiendo o que han surgido en la coyuntura actual del proceso de desarrollo capitalista proporcionan una medida de esperanza para el surgimiento de una alternativa potencial al capitalismo. En América Latina, por ejemplo, los pueblos con un reclamo legítimo de territorio participan activamente, en palabras de David Barkin (2019), «en procesos concertados para consolidar una constelación diferente de sociedades al margen del sistema capitalista global». Y en tal sentido, encontramos algunos casos de «islas de vida no capitalista».

\section{Hibridaciones de China}

A primera vista, China puede ser un lugar extraño para buscar alternativas al capitalismo o al poscapitalismo, ya que se ha descrito ampliamente como un cambio inexorable hacia el capitalismo global y su integración a éste en las últimas tres décadas. Este giro hacia el capitalismo ha generado sus fuerzas de resistencia por parte de los trabajadores, incluidos los trabajadores migrantes, así como el campesinado (véase, por ejemplo, Fan, 2018; Bieler y Lee, 2019; Wu Zhang, 2015). Que China representa una forma de capitalismo es claro. Pero quedan dos razones por las cuales China plantea 
preguntas interesantes y difíciles para una consideración de alternativas poscapitalistas. La primera se refiere a las posibilidades de «recuperación» económica, la segunda a la naturaleza y las implicaciones de las relaciones de propiedad. Ambas han sido desafíos para la periferia: ¿cómo pueden los países cerrar la brecha con los niveles de bienestar material que se encuentran en los países industrializados y qué formas de relaciones de propiedad (no capitalistas) podrían ser consistentes con esto? Estas fueron, por supuesto, preocupaciones centrales de los países «socialistas estatales» del siglo XX, pero su desaparición no hace que los desafíos sean menos relevantes para el futuro.

Con respecto a la primera pregunta, a pesar de sus muchas fallas, el capitalismo ha demostrado ser un sistema dinámico capaz de elevar el nivel de vida de una minoría de la población mundial. Una pregunta central en los ECD es cómo tales niveles de vida podrían alcanzarse de manera más amplia. Al abordar esto, el papel del Estado, y más específicamente, los usos del poder del Estado, se ha visto como esencial. Esto se ha cristalizado en torno a la idea de un Estado de desarrollo, un Estado capaz de lidiar con la tarea de emprender las múltiples transformaciones necesarias para elevar rápida y decisivamente los estándares de vida en masa. En el caso soviético, esto fue encapsulado por la frase "poder soviético más electrificación». Este modelo no tuvo éxito, pero pocos lo han logrado. Muchos países han abordado el problema central de cómo alcanzar el nivel de vida de los países industriales mediante el ejercicio del poder estatal, pero sólo un pequeño número lo ha respondido con éxito. Hay quienes, por supuesto, argumentan que es un objetivo incorrecto buscarlo. La crisis ecológica global, el sobreconsumo privilegiado de la minoría global y el trabajo pesado de la industrialización son razones por las cuales otros países no deberían 
tratar de emular el camino del centro capitalista, incluso si lo intentan por medios más humanos. Se debe imaginar un futuro diferente en el que las crisis de la modernidad capitalista sean reemplazadas por nuevas formas de solidaridad y dignidad humana; de hecho, esta es la premisa de algunas de las visiones discutidas en la siguiente sección.

Pero un aspecto del pensamiento de los ECD no es tan rápido como para abandonar el papel del Estado. El problema persistente de la desigualdad global no desaparecerá y las visiones globalistas poscapitalistas utópicas confrontan el hecho histórico de que no más de un puñado de países han hecho una transición exitosa a los niveles de vida del centro capitalista y ninguno lo ha hecho sin que el Estado sea el vehículo institucional central para su logro. A partir del reconocimiento de un mundo desigual, el potencial del Estado sigue siendo una preocupación clave para los ECD. Como escribe Katz (2018:446), una lección importante que se puede extraer de los desarrollos políticos en el siglo XX es que tanto el capitalismo como el socialismo requieren de la agencia del Estado nación. En ausencia de una redistribución global en una escala hasta ahora inimaginable, es probable que el Estado siga siendo central en los esfuerzos para cerrar las brechas en los niveles de vida entre el Norte global privilegiado y la mayoría de la población mundial en el Sur global.

Al aceptar este punto de partida, los ECD se enfrentan al incómodo hecho de que el puñado de Estados de desarrollo exitoso (del Este asiático) no ha sido democrático ni no capitalista. El éxito del modelo del Este asiático es bien conocido, pero en muchos aspectos el papel del Estado se ve, principalmente, como un administrador económico efectivo, una institución con la capacidad organizativa para efectivamente «obtener precios incorrectos» (get prices wrong), en la famosa frase de Amsden (1992). 
La reorganización del capital global en cadenas de valor globales/redes de producción global ha arrojado dudas sobre si los Estados nacionales pueden seguir este camino por más tiempo, pero nuevamente se trata de cómo gestionar la inserción en el capitalismo global. Los ECD aceptan el papel del Estado como administrador, pero lo colocan dentro del contexto del Estado como un lugar de poder. Es decir, el poder estatal se ejerce con un propósito y con consecuencias de clase y género; la exclusión y supresión del trabajo organizado en los exitosos Estados de desarrollo capitalistas, junto con su inclusión del trabajo de baja remuneración, típicamente femenino, significa que su camino no se considera deseable ni una alternativa a nada más que un camino capitalista neoliberal. Es por esta razón que continúa la búsqueda de un modelo de Estado de desarrollo democrático tanto en la teoría (White, 1998) como en la práctica (véase, por ejemplo, los debates contemporáneos en Sudáfrica).

Dentro de esta línea de ECD, China no es más que el último en la línea de los Estados en desarrollo del Este de Asia con el «ascenso» chino, aunque todavía con niveles modestos de PIB per cápita y muy lejos de los de los países capitalistas centrales, compartiendo muchas de las características de sus precursores estatales del desarrollo capitalista de Asia oriental: trabajo suprimido, empleo de género y políticas autoritarias junto con la aplicación de un conjunto bastante típico de políticas económicas estratégicas (su apertura a la inversión extranjera es una notable excepción al «paquete estándar»). Por lo tanto, en muchos sentidos, China nos lleva a enfrentar el viejo desafío de cómo elevar el nivel de vida en la periferia de manera que no dependa de la brutalidad del Estado capitalista de desarrollo, la única configuración institucional que ha demostrado ser históricamente capaz de realizar esa tarea hasta ahora. Por lo tanto, los 
ECD contienen un capítulo que considera a la agencia estatal como central para la transformación económica y social. Sin embargo, lo que los ECD no han resuelto es cómo se puede capturar o reconfigurar el Estado para facilitar un camino progresista hacia la «puesta al día» de la economía. En esto, China ofrece pocas esperanzas y está plagada de sus propias formas de crisis económica, social, política y ecológica.

Sin embargo, dentro de la experiencia china, también podemos encontrar algunas nuevas hibridaciones cuando se trata de otra cuestión central, la de los derechos de propiedad. Mientras que para muchos, China está en una transición clara hacia el capitalismo, aunque con debates sobre qué variedad de capitalismo (véase por ejemplo Naughton y Tsia, 2015; McNally, 2012; Zhang y Peck, 2014) también es posible ver la economía política de China como una forma híbrida que (todavía) no se ajusta totalmente a los dictados y las lógicas del capitalismo. En parte, esto es el resultado de la dependencia de la ruta y los elementos residuales del modelo maoísta que continúan existiendo. Cualquier sociedad alternativa o poscapitalista necesita encontrar formas de ir más allá de la propiedad privada. En el sector rural de China, el proceso de remover al campesinado de la tierra para formar un proletariado sigue siendo hasta cierto punto incompleto. Arrighi identificó este aspecto de la hibridación — la continuada propiedad colectiva de la tierra - como una razón para argumentar que «a pesar de la expansión de los intercambios de mercado en la búsqueda de ganancias (...) la naturaleza del desarrollo en China no es necesariamente capitalista» (2009:24).

¿Qué elementos de las relaciones de propiedad «no necesariamente capitalistas» podrían encontrarse en China que podrían informar futuras transiciones? Ireland y Meng (2017) han argumentado que el sector rural muestra una forma híbrida de derechos de propiedad que es consisten- 
te con las delineaciones de Marx para las relaciones de propiedad poscapitalistas (véase también Meng, 2019). Argumentan que para Marx, las sociedades poscapitalistas abolirían la propiedad privada capitalista, pero que otra propiedad privada individual (no capitalista) podría coexistir con formas colectivas y también «dentro de las estructuras de derechos de propiedad a través de una fragmentación del paquete de derechos de propiedad» (Ireland y Meng, 2017:372, énfasis en original). Esto, argumentan, es exactamente lo que se encuentra en el Sistema de Responsabilidad del Hogar rural (HRS por sus siglas en inglés) de China, donde algunas partes del paquete de derechos de propiedad (como decisiones sobre qué cultivar, qué insumos usar y flujos de ingresos derivados del uso de la tierra) se mantienen en privado, mientras que otros (como el derecho a reasignar activos) se mantienen colectivamente. Además, argumentan que el HRS, con su sistema híbrido de derechos de propiedad, fue responsable de aumentos dramáticos en la productividad agrícola y los ingresos, «así como la mejora de la autonomía y la dignidad individual» (Ireland y Meng, 2017:21). Para Ireland y Meng (2017), por lo tanto, a nivel teórico, el HRS señala las posibilidades de derechos de propiedad maleables en sociedades poscapitalistas que no se basan en la propiedad privada o estatal. Por supuesto, en la práctica, la China rural también ha experimentado expropiaciones forzadas y uso ilegal de la tierra rural, ya que los autores reconocen que podemos agregar sesgos de género (Hare et al., 2007). Sin embargo, incluso en el caso de la expropiación de tierras, no todo ha sido un proceso de «acumulación por despojo».

En este sentido, Zhan (2019) ha argumentado que al menos una parte de la expropiación ha tenido lugar sin desposesión. Como ejemplo de una serie de formas que esto ha tomado, consideremos el caso de la tierra expropiada para uso de los municipios a fin de crear industrias rurales en 
la década de 1980. Aquí Zhan argumenta que a cambio de renunciar a sus derechos de uso de la tierra, los campesinos recibieron empleos seguros en las industrias rurales recién creadas, trabajos que generalmente preferían. Mecanismos compensatorios como éstos se debilitaron sustancialmente en la década de 1990, aunque regresaron en cierta medida como resultado de las protestas campesinas en la década de los 2000 (Zhan, 2019). El punto nuevamente es mostrar que son posibles múltiples caminos hacia la industrialización con el derecho a la tierra, un legado del periodo colectivo socialista, importante para determinar los resultados para los campesinos. La cuestión de los derechos de propiedad es fundamental para las poblaciones rurales en todas partes, con los beneficios de la «propiedad grupal» analizada en India por Agarwal (2018), por ejemplo, y donde las experiencias latinoamericanas, discutidas en la siguiente sección, también son instructivas.

Por supuesto, la propiedad per se no es la variable crítica, pero sí cambia la dinámica del sistema, entonces podríamos argumentar que el capitalismo ha sido, si no reemplazado, al menos transformado en otra cosa. A este respecto, Ireland y Meng (2017) sostienen que el conjunto difuso de derechos de propiedad bajo el HRS ha actuado como un freno en la aparición de una «dinámica totalmente capitalista», un resultado que consideran deseable.

Sin embargo, Smith (2017) llega a una conclusión bastante diferente sobre los beneficios de las hibridaciones de los derechos de propiedad de China en su análisis de si China puede resolver su (y nuestro) colapso ecológico. Al dar su respuesta, argumenta que el sector industrial de propiedad estatal está libre de las restricciones normales de autorregulación del capitalismo, y que la ausencia de una dinámica capitalista conduce a resultados aún menos deseables. Smith argumenta que «en el capitalismo hay 
un límite (sic) incorporado, aunque temporal, al crecimiento: las ganancias. Si las empresas no pueden obtener ganancias, cesarán la producción y despedirán trabajadores, a veces masas de trabajadores. De vez en cuando, la recesión económica o el colapso detienen el crecimiento, al menos temporalmente, hasta que se destruya el valor suficiente para que el ciclo pueda comenzar de nuevo a mayor escala. De ahí el ciclo económico. Además, en las democracias capitalistas, todavía hay cierta libertad para organizarse, por lo que las organizaciones ambientales han podido imponer cierta restricción a la contaminación, ganancias que, como sabemos, ahora están bajo un asalto sin precedentes».

Pero, agrega Smith, la mayor parte de esto no se aplica en la economía del sector estatal de China porque los gobernantes de China no son capitalistas privados sino que «son colectivistas burocráticos que dirigen una economía capitalista híbrida burocrática-colectivista, un sistema en gran parte, aunque, por supuesto, no del todo «exenta de las leyes del capitalismo». Entonces, aquí tenemos una forma de capitalismo — capitalismo «burocrático-colectivista híbrido» que está «en gran medida exento» de las «leyes del capitalismo». La razón es que, según Smith, la economía de China no está impulsada por la maximización de ganancias sino por el objetivo de «maximizar la seguridad, el poder y la riqueza de la burocracia del Partido Comunista Chino (PCCh)». Este conductor, señala Smith, no es automático como el motor de la competencia en el capitalismo. En la economía estatal de China, el crecimiento está impulsado por las decisiones conscientes de las autoridades del partido: «Si los líderes eligen desarrollar (o no desarrollar) una industria, se desarrollará (o no). La planificación central reemplaza la competencia del mercado para dar forma al desarrollo económico». Esta propuesta de que la economía no está sujeta a las restricciones de ganancias 
aplicadas bajo el capitalismo, de hecho, se remonta al análisis de Kornai (1992) de las sociedades socialistas estatales que operan con «restricciones presupuestarias suaves». En manos de Smith, las restricciones presupuestarias no sólo conducen a la acusación de ineficiencia económica de Kornai sino a la destrucción ecológica.

La experiencia de desarrollo de China, por lo tanto, plantea preguntas importantes. Una es que abordar las desigualdades globales entre países sigue siendo una preocupación central e ineludible, pero los Estados de desarrollo capitalistas que han tenido cierto éxito en el cumplimiento de este objetivo (limitado) no han sido progresistas en el sentido de que promovieron la democracia, la equidad o la libertad, y China no es la excepción. En segundo lugar, las formas de propiedad híbrida que representan un alejamiento de las relaciones de propiedad capitalistas son posibles pero que son tan capaces de intensificar las crisis como estimular la esperanza. No hay un final de cuento de hadas, sino una lucha continua en China como en otros lugares sobre el papel del Estado, las formas de relaciones de propiedad y la formación y resistencia de clase.

\section{Las hibridaciones de América Latina}

Un principio fundamental de la economía política marxista, es decir, el materialismo histórico, es que el progreso en el desarrollo de las fuerzas productivas, y cada avance en la marcha del capital, produce un cambio correspondiente en las relaciones de producción, así como en las fuerzas de resistencia y la lucha de clases asociada con estas fuerzas. Este principio se ilustra claramente en los ciclos de desarrollo y resistencia que se han desarrollado en 
la periferia latinoamericana del sistema mundial en el periodo de desarrollo capitalista posterior a la Segunda Guerra Mundial. Aquí es posible trazar los contornos de tres de estos ciclos, el primero asociado con la era del Estado desarrollista (entre los 1950 y 1970); el segundo se relaciona con la era neoliberal asociada a la instalación de un nuevo orden mundial diseñado para liberar el capital y las llamadas «fuerzas de libertad económica» (capitalismo, mercado, empresa privada) de las restricciones regulatorias del Estado desarrollista; el tercer ciclo está asociado con la expansión del capital «extractivo» que busca recursos (invertido en la extracción de riqueza de recursos naturales y su exportación en forma de productos primarios) y el surgimiento de China y la reconfiguración del poder económico mundial.

América Latina es un laboratorio importante y útil analíticamente para estudiar la dinámica de estos tres ciclos de resistencia al desarrollo; esto se debe principalmente a que en ninguna otra región la agenda de política neoliberal —una fuerza impulsora en el proceso de desarrollo capitalista— se ha implementado de manera tan completa y con un impacto tan devastador en las fuerzas productivas existentes tanto en la agricultura como en la industria; y en ninguna otra macrorregión las fuerzas de resistencia han sido tan efectivas, generando no sólo movimientos sociales poderosos para movilizar las fuerzas de resistencia, una lucha de clases desde abajo, sino una serie de alternativas teóricamente definidas y construidas políticamente formas de desarrollo alternativas (posneoliberales) y alternativas al desarro1lo (capitalista) (Munck y Delgado, 2018).

Estas propuestas de modelos alternativos de desarrollo, alternativas tanto al neoliberalismo como al capitalismo, se elaboraron en el contexto de un «ciclo progresista» en la política latinoamericana (2002-2015) asociado con el giro de los gobiernos en América del Sur —incluida Argentina, 
Brasil, Bolivia, Ecuador y Venezuela — hacia el «activismo estatal inclusivo», el «neodesarrollismo» y el «neoextractivismo» (utilizando las rentas de recursos y los ingresos de las exportaciones de productos básicos para financiar programas de reducción de la pobreza). Sobre la dinámica económica y política de este «ciclo progresista», véase, entre otros, Veltmeyer y Petras (2014).

En este contexto, las propuestas alternativas «desde abajo» (dentro de las comunidades afectadas negativamente por el avance del capital extractivo y los movimientos sociales formados para movilizar las fuerzas de resistencia) han asumido tres formas básicas: a) un modelo de posdesarrollo (Vivir Bien o Buen Vivir) elaborado sobre la base de una cosmovisión indígena sobre cómo los humanos pueden «vivir bien» en solidaridad social y armonía con la naturaleza (Acosta, 2012); b) un modelo de desarrollo local basado en la comunidad mediante el cooperativismo y la autogestión de los trabajadores, así como una cultura de solidaridad social; este modelo ha tomado la forma predominante de experimentos con la construcción de una «economía social y solidaria» (ESS); y c) un modelo diseñado para encontrar un camino hacia el «socialismo del siglo XXI» desplegado en Venezuela (Katz, 2018). Analizamos los dos primeros de estas formas más abajo.

\section{Buen Vivir como posdesarrollo}

El concepto de Buen Vivir o Vivir Bien está incrustado en una cultura indígena de solidaridad y una concepción de los humanos como parte de la naturaleza y como guardianes de un medioambiente saludable y los derechos de la naturaleza (Acosta, 2012). Estos últimos han sido consagrados en nuevas constituciones políticas del Estado plurinacional y multiétnico promulgado 
en Bolivia y Ecuador por regímenes «progresistas» con su base social en las comunidades y nacionalidades indígenas del país (Acosta, 2012).

En cuanto al modelo de esta forma de posdesarrollo, dado el debate inestable sobre la estrategia neoextractivista de desarrollo nacional adoptada por ambos regímenes - una estrategia que ha sido fuertemente criticada por las comunidades indígenas y los economistas e intelectuales de izquierda-, aún no ha tomado una forma definitiva. Más bien, los propulsores de la transformación sistémica continúan debatiendo las características contradictorias de una estrategia y políticas que se basan en la agencia del capital extractivo: inversión extranjera directa en la adquisición de tierras a gran escala y la extracción de recursos naturales que se exportan en una forma y bajo condiciones que conllevan superganancias para el capital global, costos socioambientales excesivamente altos a cargo de las comunidades más directamente afectadas por las operaciones destructivas del capital extractivo y la violación de los derechos territoriales de estos miembros de la comunidad, así como los derechos de la naturaleza (Gudynas, 2013; Veltmeyer y Petras, 2014).

Como Barkin (2019) ha argumentado, la lucha de las comunidades indígenas en la frontera del capital extractivo no debe verse tanto como una lucha de clases por la tierra y el trabajo como una lucha territorial ${ }^{1}$ —una

\footnotetext{
${ }^{1} \mathrm{Al}$ debatir sobre los «bienes comunes globales», se debe hacer una distinción entre «tierra», que, según Walter Barraza, un miembro representativo del pueblo Tonocoté, el camache (jefe) del pueblo Tonocoté de la provincia de Santiago del Estero, Argentina, «se relaciona con la propiedad privada» como un «concepto capitalista», mientras que «territorio incluye (...) personas que viven en ese lugar (...) [con la] obligación de cuidar su naturaleza». Añade que «nosotros los pueblos nativos vivimos en armonía con nuestros hermanos animales, plantas, agua. Somos parte del territorio, lo que nos proporciona todo lo que necesitamos. Talar bosques es como cortar una rama. Vienen por los recursos naturales, mientras vivimos en armonía con esos recursos (Pedrosa, 2017).
} 
lucha de estas comunidades para reclamar sus derechos territoriales y los derechos de la naturaleza-, una lucha fundamentalmente informada por una concepción indígena de un universo alternativo, una realidad alternativa u ucronía incrustada en la noción Buen Vivir. En términos de esta ucronía, las comunidades indígenas y campesinas de América están reestructurando sus organizaciones y estructuras de gobernanza, tomando el control de los territorios que han reclamado por generaciones. Según lo documentado por Barkin, además de movilizar sus fuerzas de resistencia contra las operaciones del capitalismo extractivo, estas comunidades, que Barkin concibe como un «sujeto revolucionario colectivo», también están «reorganizando la producción [para] generar un excedente, reuniendo sus miembros para aprovechar los recursos subutilizados y las energías de las personas para mejorar su capacidad de elevar el nivel de vida y asegurar la conservación y restauración del medioambiente».

Estas comunidades no operan de manera aislada. Coordinan actividades, comparten información y crean alianzas (Abya Yala, 2009). De hecho, Barkin (2019) argumenta que cientos de millones de personas están participando en este creciente movimiento; además, existe un gran potencial para que otros se unan a ellos, expandiéndose desde las áreas sustanciales donde ya están operando y formando alianzas y redes sociales regionales y globales para garantizar que esta dinámica de cambio social pueda crecer.

\section{Construyendo una economía social y solidaria}

Como han señalado Barkin y Sánchez (2017), en toda América Latina hoy en día las personas están organizando formas alternativas de asegurar sus medios de vida y comunidades basadas en el desarrollo local, el intercambio 
social y el cooperativismo, el desarrollo local o la economía ecológica, ya sea construida «desde abajo» (es decir, desde dentro de la comunidad) o en la búsqueda de una estrategia diseñada por agentes de cooperación internacional (agencias de desarrollo, como el BM) como una forma de aliviar las presiones sobre un mercado laboral urbano saturado o disfuncional o gobiernos que buscan gestionar las demandas y necesidades de una creciente población urbana excedente.

En estas diversas acciones y experimentos colectivos, hay dos concepciones fundamentalmente diferentes de una economía social y solidaria: una avanzada como parte de una estrategia para gestionar las dinámicas complejas del desarrollo urbano y rural, la otra como parte de una estrategia de base y movimiento social por confrontar lo que los zapatistas llaman la «hidra capitalista» (EZLN, 2015) y encontrar alternativas que ofrezcan más oportunidades y una mejor calidad de vida que la que ofrece la economía capitalista actual. La idea aquí es que las diversas estrategias desarrolladas por las organizaciones y fuerzas internacionales involucradas en el proyecto de cooperación y desarrollo internacional, incluida una estrategia de desarrollo local, basado en la comunidad y en el empoderamiento de los pobres (movilizando su capital social para el autodesarrollo, para que «posean» su propio desarrollo), están diseñadas como mecanismos de ajuste que son funcionales para la continuación del capitalismo. Sin embargo, desde una perspectiva izquierdista, esta estrategia puede verse como una táctica neoliberal para defender el sistema de las fuerzas de resistencia, una forma híbrida bastarda de «desarrollo local».

Por el contrario, en el contexto latinoamericano, la construcción de una economía social se ha asociado estrechamente con diversos experimentos con el movimiento cooperativo y diversas experiencias con 
fábricas de autogestión y gestión de trabajadores, basadas en consejos de trabajadores y asambleas de fábricas que surgieron en el contexto más amplio de lo que podría describirse como una lucha de clases (Azzellini, 2017). Esto es evidente en las tres fases del desarrollo capitalista de posguerra.

Por ejemplo, en Chile bajo el régimen socialista de Salvador Allende (1970-1973), más de 125 fábricas cayeron bajo algún sistema de autogestión de los trabajadores. Alrededor de la mitad de estas fábricas eran controladas por oficiales públicos o funcionarios, lo que representa una forma parlamentaria de gobierno con sus divisiones políticas de izquierda-centro-derecha; la otra mitad estaba a cargo de comisiones de trabajadores a nivel de fábrica que se organizaron siguiendo los lineamientos de los consejos de trabajadores o sóviets, al igual que la Comuna de París de Francia a mediados del siglo XIX. Y luego tenemos a Argentina en el contexto de la crisis de 2001-2002, cuando los trabajadores ocuparon y se hicieron cargo de más de 200 fábricas, defendiéndolas del cierre, protegiendo el empleo de los trabajadores y mejorando de manera considerable las condiciones sociales de trabajo y, lo más importante, elevando conciencia política de los trabajadores. La mayoría de estas fábricas, empresas recuperadas por sus trabajadores (ERT), como algunos las han denominado, se organizaron como cooperativas o colectivos de trabajadores autogestionados por los trabajadores (Azzellini, 2017). También podemos citar la experiencia de Bolivia a raíz de la Revolución de 1952, y Perú en 1967, cuando un grupo de militares progresistas nacionalistas liderados por el general Velasco Álvarez tomó el poder y expropió una gran cantidad de minas/fábricas/plantaciones, estableciendo un sistema de cooperativas industriales (y comunidades) basado en la toma conjunta de decisiones por parte de la gerencia y los trabajadores. 
El caso de Brasil es muy importante, aunque rara vez se discute en la literatura. Actualmente, más de 200 empresas han sido «recuperadas» por los trabajadores, con la primera experiencia en la fábrica de zapatos Makerli en 1991. La Asociación Nacional de Trabajadores en Empresas Autogestionadas (Anteag) se estableció en 1994 con el objetivo de coordinar los diversos proyectos que se materializaron en condiciones de crisis y casi colapso de la industria. Tiene oficinas en seis estados a cargo de acompañar proyectos de autogestión, buscando la integración con organizaciones no gubernamentales (ONG), gobiernos estatales y municipales. La Anteag ve la autogestión como un modelo organizacional que combina la propiedad colectiva de los medios de producción con la autonomía de la toma de decisiones empresariales y la participación activa en la gestión democrática.

El punto importante sobre éstos y otros experimentos similares con el cooperativismo y el sindicalismo, y la construcción de una economía social y solidaria basada en el desarrollo local y en la comunidad, es que deben entenderse en el contexto más amplio del desarrollo capitalista, la historia del capitalismo en la región.

\section{Conclusiones}

Para los pueblos y países en la periferia del sistema capitalista global, los desafíos del futuro incluyen algunos familiares, a saber, cómo cerrar la brecha en los niveles de vida con los países industrializados y cómo compartir los recursos e ingresos del mundo de manera más equitativa dentro y entre países. A estos desafíos familiares se puede agregar el nuevo de destrucción ecológica. Los ECD argumentan que tales desafíos no pueden 
entenderse sin analizar la dinámica sistémica y las contradicciones fundamentales del capitalismo, y que es probable que se encuentren alternativas al capitalismo en la periferia y no en el centro del sistema mundial.

Al examinar las experiencias recientes y la dinámica de desarrollo de China y América Latina, hemos resaltado una serie de hibridaciones que pueden informar cómo pueden evolucionar las alternativas al capitalismo. Por ejemplo, las formas de propiedad híbrida en la China rural ofrecen alguna evidencia de cómo se podrían transformar las relaciones de propiedad capitalistas; los experimentos latinoamericanos en la construcción de una economía social y solidaria también muestran cómo se podrían constituir las relaciones de propiedad poscapitalistas y el desarrollo participativo (toma de decisiones verdaderamente democrática); las poderosas fuerzas de resistencia al avance del capital en la frontera extractiva que han surgido en América Latina han allanado varias formas de ir más allá del capitalismo en el siglo XXI.

Las comunidades indígenas han sido pioneras en una forma en la frontera extractiva que participan en una lucha territorial para reclamar su acceso a los bienes comunes mundiales de la tierra, el agua y sus derechos territoriales. La clave de esta lucha es una concepción indígena de cómo vivir bien en solidaridad social y armonía con la naturaleza. El problema con este enfoque, sin embargo, es cómo ampliar el proceso de transformación productiva y social al nivel de desarrollo nacional. Dos gobiernos en América Latina (Ecuador, Bolivia) han asumido el desafío, pero en el contexto de un sistema mundial dominado por el capital, y las presiones sobre ellos para integrarse en este sistema a través de un proceso de globalización, ambos gobiernos se vieron obligados a desarrollar un forma híbrida de posdesarrollo, que combina una concepción indígena de Vivir Bien (Buen Vivir) 
con el neodesarrollismo (la búsqueda de una forma de desarrollo más inclusiva) y el neoextractivismo (utilizando los ingresos de las exportaciones comunitarias para financiar un proceso de reducción de la pobreza). Sin embargo, parece que este modelo híbrido, dependiente de un ciclo de altos precios de las exportaciones de productos básicos, ha fallado y se ha hundido en el arrecife del desarrollo capitalista.

Otra vía diferente de desarrollo alternativo y cambio transformador ha sido pionera en Venezuela: «el socialismo del siglo XXI», avanzado con la agencia no sólo del Estado sino también con organizaciones de base en las comunidades. Sin embargo, esta forma híbrida de socialismo con capitalismo también ha sido víctima no sólo de las fuerzas del imperialismo estadounidense, sino también de varios problemas y contradicciones del capitalismo extractivo (como una dependencia excesiva en la extracción de recursos naturales) (véase Petras y Veltmeyer, 2019).

En el mejor de los casos, los diversos experimentos y experiencias latinoamericanas con el «socialismo» y las formas alternativas de «posdesarrollo» ofrecen vislumbres de lo que podría ser posible. Pero en tiempos de crisis capitalistas múltiples y profundas, es importante vislumbrar alternativas.

\section{Referencias}

Abya Yala (Movimientos Indígenas, Campesinos y Sociales) (26 de febrero de 2009), «Diálogo de alternativas y alianzas», Minga Informativa de Movimientos Sociales, La Paz, México.

Acosta, Alberto (2012), Buen Vivir. Sumak kawsay. Una oportunidad para imaginar otros mundos, Quito, AbyaYala. 


\section{PAul Bowles | HenRy VeltMeyer}

Agarwal, Bina (2018), «Can group farms outperform individual family farms? Empirical insights from India», World Development, 108, pp. 57-73.

Amsden, Alice (1992), Asia's next giant: South Korea and late industrialization, Oxford, Oxford University Press.

Arrighi, Giovanni (2009), Adam Smith in Beijing: lineages of the 21st Century, London, Verso.

Azzellini, Dario (2017), Communes and workers' control in Venezuela: building 21st Century socialism from below, Leiden, Brill.

Banco Mundial (BM) (s/f), «About the Worl Bank», en https://www.worldbank. org/en/about

Barkin, David (junio de 2019), «The communitarian revolutionary subject: new forms of social transformation», Third world Quarterly.

Barkin, David y Alejandra Sánchez (septiembre de 2017), «The collective revolutionary subject: new forms of social transformation», unedited paper for Revolutions: A Conference, Winnipeg.

Bieler, Andreas y Chun-Yi Lee (2019), Chinese labour in the global economy: capitalist exploitation and strategies of resistance, London: Routledge.

Ejército Zapatista de Liberación Nacional (EZLN) (2015), El pensamiento crítico frente a la hidra capitalista (volumen 1), en http://enlacezapatista.ezln.org. mx/2015/07/13/indice-volumen-uno-participaciones-de-la-comision-sextadel-ezln-en-el-seminario-el-pensamiento-critico-frente-a-la-hidra-capitalista

Fan, Shigang (2018), Striking to survive: workers' resistance to factory relocations in China, Chicago, Haymarket Books.

Foster, John Bellamy (2002), «Capitalism and ecology. The nature of the contradiction», Monthly Review, 54(04), pp. 6-16.

Foster, John Bellamy, Brett Clark y Richard York (2010), The ecological rift. Capitalism's war on the earth, New York, Monthly Review Press. 


\section{Alternativas de desarrollo: Viejos desafíos}

Gudynas, Eduardo (2011), «Buen Vivir: today's tomorrow», Development, 54(4), pp. 441-447.

(2013), «Debates on development and its alternatives in Latin America: a brief heterodox guide», en Miriam Lang, Lyda Fernando y Nick Buxton (eds.), Beyond development: alternative visions from Latin America, Amsterdam, Transnational Institute.

Hare, D., Yang Li and Englander, D., (2007), «Land Management in Rural China and its Gender Implications,» Feminist Economics, 13(3-4): 35-61.

Ireland, Paddy y Gaofeng Meng (2017), «Post-capitalist property», Economy and Society, 46(3), pp. 369-397.

Katz, Claudio (2018), «Socialism and development: a Latin American perspective», en Henry Veltmeyer y Paul Bowles (eds.), The essential guide to critical development studies, London, Routledge, pp. 435-450.

(2018), «Development theory: the Latin American pivot», en Henry

Veltmeyer y Paul Bowles (eds.), The essential guide to critical development studies, London, Routledge, pp. 73-83.

Kornai, Janos (1992), The socialist system: the political economy of communism, Princeton, Princeton University Press.

McNally, Christopher (2012), «Sino-capitalism: China's reemergence and the international political economy», World Politics, 64(4), pp. 741-776.

Meng, Gaofeng (2019), «The household responsibility system, Karl Marx’s theory of property and Antony M. Honoré's concept of ownership», Science \& Society, 83(3), pp. 300-326.

Munck, Ronaldo y Raúl Delgado Wise (2018), Rethinking Latin American development, London, Routledge.

Naughton, Barry y Kelle S. Tsai (2015), State capitalism, institutional adaptation and the Chinese miracle, Cambridge, Cambridge University Press. 


\section{PAul Bowles | HenRy VeltMeyer}

Pedrosa, M. (8 de septiembre de 2017), «Argentina: native communities face eviction, death of protective law is not renewed by Congress», Tiempo Argentino. Petras, James y Henry Veltmeyer (2013), Imperialism and capitalism in the Twenty-first Century: a system in crisis, Franham, Ashgate. (2019), Latin America in the vortex of social change, London, Routledge.

Programa de las Naciones Unidad para el Desarrollo (PNUD) (s/f), «Sustainable development goals», en https://www.undp.org/content/undp/en/home/sustainable-development-goals.html

Smith, Richard (2017), «China's drivers and planetary ecological collapse», Real-World Economics Review (82).

Veltmeyer, Henry y Paul Bowles (2018), The Essential Guide to Critical Development Studies, London, Routledge.

(2014), The new extractivism: a model for Latin America?, London, Zed Books.

Veltmeyer, Henry y Raúl Delgado Wise (2018), Critical development studies: an introduction, Halifax, Fernwood publications.

White, Gordon (1998), «Building a democratic developmental state: social democracy in the developing world», Democratization, 5(3).

Zhan, Shaohua (2019), «Accumulation by and without dispossession: rural land use, land expropriation and livelihood implications in China», Journal of Agrarian Change, 19, pp. 447-464.

Zhang, Jun y Jamie Peck (2016), «Variegated capitalism, Chinese style: regional models, multi-scalar constructions», Regional Studies, 50(1), pp. 52-78.

Zhang, Wu, (2015), «Leadership, Organization and Moral Authority: Explaining Peasant Militancy in Contemporary China», The China Journal, 73, pp.59-83. 